\title{
Adding value to the diagnostic process
}

\author{
Laurien Kuhrij (D) , ${ }^{1}$ Perla J Marang-van de Mheen (D) ${ }^{2}$
}

${ }^{1}$ Department of Biomedical Data Sciences, Leiden University Medical Center, Leiden, Netherlands

${ }^{2}$ Department of Biomedical Data Sciences, Leids Universitair Medisch Centrum, Leiden, Netherlands

\section{Correspondence to}

Dr Laurien Kuhrij, Department of Biomedical Data Sciences, J10-S, Leiden University Medical Center, Leiden, The Netherlands; L.S.Kuhrij@lumc.nl

Accepted 16 November 2021

\section{SLinked}

http://dx.doi.org/10.1136/ bmjqs-2021-013672

Check for updates

(c) Author(s) (or their employer(s)) 2021. No commercial re-use. See rights and permissions. Published by BMJ.

To cite: Kuhrij L, Marang-van de Mheen PJ. BMJ Qual Saf Epub ahead of print: [please include Day Month Year]. doi:10.1136/

bmjqs-2021-014092
In 2010, Porter introduced the concept of value-based healthcare in an attempt to shift the focus from volume of services to value created for patients. ${ }^{1}$ Value was defined based on important health outcomes achieved for patients relative to costs, rather than being based on the input in terms of volume of delivered services. Since multiple professionals and organisational units are involved in healthcare, the idea was that all stakeholders should work towards a shared goal of creating value in improving health outcomes that meet the needs of the patient. Besides health outcomes, Porter included time to achieve recovery and return to normal activities, and disutility in care or treatment processes as important outcomes related to the recovery process. The diagnostic process is mentioned as part of this recovery process, by referring to delays in diagnosis and diagnostic errors as possible disutility of care, rather than as a process that may add value. In contrast, radiologists from multiple professional societies have recently argued that the diagnostic process should be considered as potential loss of value and as an intrinsic value creator. ${ }^{2}$ Based on this perspective, every step in the diagnostic chain potentially creates value as it influences subsequent decision making to contribute to improving the relevant outcome(s) for the patient.

Many specialties have embraced Porter's value-based healthcare framework, reporting on application in a comprehensive outcome set and implementation of these principles. ${ }^{3}$ However, the university of Utah in 2017 published the sobering results of the Value in Health Care survey, where they asked 5031 patients, 687 physicians and 538 employers how they defined value in healthcare and whom they felt was responsible for achieving it. ${ }^{4}$ The results showed a complete disconnect between patients and physicians when asked to choose among five statements that reflected what they value most in healthcare: $90 \%$ of patients selected different combinations of value statements than any combination chosen by physicians. For patients, the top value statement was 'My Out-of-Pocket Costs Are Affordable' (chosen by 45\%) and only 32\% chose 'My Health Improves', the latter even slightly below 'Staff Are Friendly and Helpful', whereas physicians mostly focused on quality and service measures and would consider clinical outcomes of paramount importance. In terms of responsibility, $75 \%$ of physicians hold themselves responsible for a patient's health improving, whereas patients assigned responsibility both to themselves (45\%) and physicians (44\%). Both types of misalignment indicate that if doctors and patients do not agree what value means, we are not going to make any progress in achieving value-based healthcare. ${ }^{5}$ To grasp the different aspects of value among different stakeholders, the European Commission has recently published a draft opinion paper, defining value-based healthcare by distinguishing four value pillars: (1) appropriate care to achieve patients' personal goals (personal value), (2) achievement of best possible outcomes with available resources (technical value), (3) equitable resource distribution across all patient groups (allocative value) and (4) contribution of healthcare to social participation and connectedness (societal value). ${ }^{6}$

\section{ERRORS IN THE DIAGNOSTIC PROCESS}

Returning to the area of diagnosis, there is an extensive literature on the frequency of errors in the diagnostic process ${ }^{7}$ and how they cause patient harm, ${ }^{8-10}$ with the recent National Academy of Medicine report concluding that most people will experience at least one diagnostic 
error in their lifetime. ${ }^{11}$ Diagnostic errors are one of the medical errors that inflict the most harm, and the most common reason for malpractice claims. ${ }^{9}$ To gain insight into what went wrong and where in the diagnostic process, this complex process has typically been characterised by analysing physician-reported errors and classifying these in a framework, where classification makes the complexity more manageable. ${ }^{12}$ The results show which specific diagnoses were missed, in what stage of the diagnostic process and their perceived impact on patient outcomes. Such studies are predicated on the assumption that a diagnostic error occurred, in which there is clear evidence of a 'missed opportunity' to make a correct or more timely diagnosis. However, these may only show part of the problem by focusing on those harmed and/or based on clinician-reported information. A different perspective-that of the patient-might give additional insight.

In this issue of BMJ Quality \& Safety, Bell and colleagues take a similar approach to that used in an earlier study by Schiff and colleagues ${ }^{12}$ in analysing what went wrong and where in the diagnostic process, but using patient reports instead of physician reports to develop a framework specifically for patientreported diagnostic process-related breakdowns in ambulatory care. ${ }^{13}$ Importantly, they focused on breakdowns rather than diagnostic errors for several reasons. First, patients may feel more comfortable in identifying a breakdown in process than an error. Second, this approach can also capture proximal failures rather than only the final harmful event. Finally, identifying upstream breakdowns may also inform prevention strategies. These patient-reported breakdowns can thereby bring a different perspective to the same types of diagnostic error already identified in clinician-reported data or add new categories of errors or breakdowns to existing (clinician-based) diagnostic error frameworks. The framework provided by Bell and colleagues includes 7 patient-reported breakdown categories (with 40 subcategories), 19 patient-reported contributing factors and 11 potential patient-reported impacts. 'Communication and respect' was added as a new category of breakdowns compared with existing frameworks on diagnostic errors derived from clinician reports, ${ }^{12}$ although this is already included as a category in the Healthcare Complaints Analysis Tool used to systematically categorise patient complaints. ${ }^{14}$ This new category of breakdowns occurred commonly-in about 30\% of patient reports-and highlighted feelings of not being heard and misalignment between patient and provider on (the significance of) symptoms and events. ${ }^{13}$ Also highlighted, although less common, were documentation issues and various technology related issues that are also included in the sociotechnical dimensions of the Safer Dx framework, ${ }^{15}$ such as poor access to patient notes that could be particularly relevant in the contexts of equity and social determinants of health. Applying the framework to analyse 2165 patientreported ambulatory errors in two surveys conducted in the USA, Bell and colleagues found the frequency of patient-reported diagnostic process-related breakdowns to be $6.4 \%$ in one survey and $6.9 \%$ in the other, with good to excellent intercoder reliability between a patient and two physician coders. Active involvement of patients in developing the framework and in the coding might be important if future tools are developed that use this framework to help patients identify breakdowns.

The examples given by Bell and colleagues of how patient reports were coded in the framework, make clear that deliberately using a broader definition and focusing on the entire diagnostic process rather than starting from a diagnostic error, provides insight into the wider consequences of breakdowns identified early in the process. However, such consequences were only coded in the impact category 'patient activation or mitigation', without further specification where or what the impact later in the process might have been if it had not been mitigated. For instance, an incorrect date for a cervical cancer screening smear affects patient follow-up, but would be coded as the breakdown occurring during 'tests and referrals' (ordering/ scheduling) with the impact coded as 'patient activation and mitigation'. As a result, the coding loses part of the relevant context that the breakdown affected the follow-up and timeliness of diagnostic screening. Coding these consequences in more detail would make it easier to use coded data to assess how one category of breakdown may have multiple consequences and vice versa. It would also show what decisions further in the process, such as regarding treatment or during follow-up, might be most affected by earlier breakdowns, thereby creating more awareness among physicians of how seemingly simple errors may have broader consequences.

What is also highlighted by these examples is that patients and clinicians may not have shared goals, particularly reflected in the finding that patients often do not feel heard. For instance, one patient's comment was 'I have a serious dental issue and cannot afford to fix it-my cardiologist and GP [General Practitioner] ignore this issue-and I mention it on a regular basis'. Both the cardiologist and GP may consider this as outside their control, focusing only on their specialty and resources (technical value), whereas for the patient, this clearly is important (personal value). The subcategory 'misalignment between patient and clinician about symptoms, events, or significance of these' may include more examples. More in-depth exploration to better understand such misalignment and explicit communication about goals of care and what is most important for the patient, may help to mitigate this gap. ${ }^{16}$ 


\section{USING THE FRAMEWORK TO ADD VALUE}

Ultimately, the diagnostic process needs to add value to the care delivered to patients. Bell and colleagues have shown that patients are able to identify breakdowns and give a more comprehensive view including the potential impact later in the process, which could be used for prevention efforts. For instance, the breakdowns stemming from copy/paste documentation could be explored to understand why there is a need for the doctor to copy/paste. Perhaps there are other ways to keep information up-to-date for example, to leverage technology to more actively engage patients in confirming whether medical history or current complaints are still relevant today, what is in the past and whether new information should be added. We let patients routinely confirm whether they still have the same GP or telephone number, so why not also do that for other information that we need to ask patients or their family? Similarly, we can explore different combinations of a specific type of breakdown with contributing factors or impact, to more selectively target areas for improvement. If such a combination occurs frequently enough, it could indicate the need for an intervention. The framework seems less useful for evaluating the impact of known ${ }^{17}$ or new interventions, or to monitor changes in breakdowns over time, due to the heterogeneity within (sub)categories. Specific interventions may only improve some types of breakdowns but do nothing to improve others within the same (sub)category of the framework. This heterogeneity combined with small numbers of specific breakdowns targeted by an intervention, will mean a poor signal-to-noise ratio to detect changes over time. $^{18}$

Rather than continuing to report frequencies of types of diagnostic errors or breakdowns, we need to move forward to improve the diagnostic process. We know the lay of the land fairly well if we combine both clinician and patient reported information; applying the existing frameworks to different populations will likely add relatively little new information. Instead, more may be gained if we start using such frameworks to create value for patients. Every step of the diagnostic chain needs to create value in order to contribute to clinical decision-making and the outcome(s) that are relevant to that patient, and future metrics should be able to capture that. ${ }^{2}$ But perhaps the most important message of Bell and colleagues' study is that we need to actively engage patients in every part of the diagnostic process. We should ask what is most important for them and how we can deliver appropriate care to achieve their personal goals, as we cannot assume to know what they value most in healthcare. ${ }^{19}$ Only then can we start adding value to the diagnostic process.

Contributors LK and PJM-vdM both contributed to conception of the paper, critically read and modified subsequent drafts and approved the final version. PJM-vdM is editor at BMJ Quality \& Safety.
Funding The authors have not declared a specific grant for this research from any funding agency in the public, commercial or not-for-profit sectors.

Competing interests None declared.

Patient consent for publication Not applicable.

Provenance and peer review Commissioned; internally peer reviewed.

\section{ORCID iDs}

Laurien Kuhrij http://orcid.org/0000-0003-0640-5431

Perla J Marang-van de Mheen http://orcid.org/0000-00031439-0989

\section{REFERENCES}

1 Porter ME. What is value in health care? N Engl J Med 2010;363:2477-81.

2 Brady AP, Bello JA, Derchi LE, et al. Radiology in the era of value-based healthcare: a Multi-Society expert statement from the ACR, CAR, ESR, IS3R, RANZCR, and RSNA. Radiology 2021;298:486-91.

3 Lobatto DJ, Zamanipoor Najafabadi AH, de Vries F, et al. Toward value based health care in pituitary surgery: application of a comprehensive outcome set in perioperative care. Eur J Endocrinol 2019;181:375-87.

4 University of Utah. Value in health care survey. University of Utah, 29th November 2017. Available: https://healthcare.utah. edu/publicaffairs/news/2017/11/value-survey.php

5 Pendleton RC. We Won't Get Value-Based Health Care Until We Agree on What "Value" Means. Harvard Business Review, 2018.

6 European Commission Expert Panel on Effective Ways of Investing in Health. Draft opinion paper on "Defining Value in 'value-based healthcare”", 2019. Available: https://ec. europa.eu/health/expert_panel/sites/expertpanel/files/024_ valuebasedhealthcare_en.pdf

7 Gunderson CG, Bilan VP, Holleck JL, et al. Prevalence of harmful diagnostic errors in hospitalised adults: a systematic review and meta-analysis. BMJ Qual Saf 2020;29:1008-18.

8 Cheraghi-Sohi S, Holland F, Singh $\mathrm{H}$, et al. Incidence, origins and avoidable harm of missed opportunities in diagnosis: longitudinal patient record review in 21 English general practices. BMJ Qual Saf 2021. doi:10.1136/ bmjqs-2020-012594. [Epub ahead of print: 14 Jun 2021].

9 Gupta A, Snyder A, Kachalia A, et al. Malpractice claims related to diagnostic errors in the hospital. BMJ Qual Saf 2018;27:53-60.

10 Winters B, Custer J, Galvagno SM, et al. Diagnostic errors in the intensive care unit: a systematic review of autopsy studies. BMJ Qual Saf 2012;21:894-902.

11 National Academies of Sciences, Engineering, and Medicine 2015. Improving diagnosis in health care. Washington, DC: The National Academies Press.

12 Schiff GD, Hasan O, Kim S, et al. Diagnostic error in medicine: analysis of 583 physician-reported errors. Arch Intern Med 2009;169:1881-7.

13 Bell SK, Bourgeois F, DesRoches CM, et al. Filling a gap in safety metrics: development of a patient-centred framework to identify and categorise patient-reported breakdowns related to the diagnostic process in ambulatory care. BMJ Qual Saf 2021. doi:10.1136/bmjqs-2021-013672. [Epub ahead of print: 16 Oct 2021].

14 Gillespie A, Reader TW. The healthcare complaints analysis tool: development and reliability testing of a method for 


\section{Editorial}

service monitoring and organisational learning. BMJ Qual Saf 2016;25:937-46.

15 Singh H, Sittig DF. Advancing the science of measurement of diagnostic errors in healthcare: the safer DX framework. BMJ Qual Saf 2015;24:103-10.

16 Giovannetti ER, Clair CA, Jennings LA, et al. Standardised approach to measuring goal-based outcomes among older disabled adults: results from a multisite pilot. BMJ Qual Saf 2021;30:157-66.
17 Dave N, Bui S, Morgan C, et al. Interventions targeted at reducing diagnostic error: systematic review. BMJ Qual Saf 2021. doi:10.1136/bmjqs-2020-012704. [Epub ahead of print: 18 Aug 2021].

18 Shojania KG, Marang-van de Mheen PJ. Identifying adverse events: reflections on an imperfect gold standard after 20 years of patient safety research. BMJ Qual Saf 2020;29:265-70.

19 Naik AD, Catic A. Achieving patient priorities: an alternative to patient-reported outcome measures (PROMs) for promoting patient-centred care. BMJ Qual Saf 2021;30:92-5. 